\title{
THE MOLECULAR AND CRYSTAL STRUCTURE OF (+)-MIANSERIN HYDROBROMIDE
}

\author{
C. VAN Ru and D. FeIL* \\ Laboratory of Chemical Physics, Twente University of Technology, P.O. Box 217, \\ Enschede, The Netherlands
}

(Received in UK 4 September 1972; Accepted for publication 16 February 1973)

\begin{abstract}
X-ray analysis of crystals of (+)-mianserin hydrobromide $(1,2,3,4,10,14 b$-hexahydro-2methyl-dibenzol $[c, f]$ pyrazino[1,2-a]azepine) has established the stereochemistry and absolute configuration of this compound ( $S$ at $14 b$ ).

The crystals are orthorhombic with unit-cell dimensions: $a=1299(1) \mathrm{pm}, b=1420(1) \mathrm{pm}, c=$ 1002 (1)pm; $Z=4$. The space group is $P 2_{1} 2_{1} 2_{1}$. Intensity data for about 1000 reflections were measured on a single-crystal diffractometer with $\mathrm{Cu}-K_{\alpha}$ radiation. The crystal structure was solved by the heavy-atom method and refined by least-squares procedures. Final $R$-factor $9.4 \%$. The absolute configuration was determined by Bijvoet's technique.
\end{abstract}

\section{INTRODUCTION}

The synthesis of mianserin (1) has recently been reported. ${ }^{1}$ Spectroscopic data on $\mathbf{1}^{2}$ are in good agreement with an anti piperazino-azepine junction and the expected chair-like piperazino ring. However, these measurements gave neither conclusive evidence as to the conformational state of the azepine (either a or b of Fig 1) nor an accurate estimate of the dihedral angle occurring between the two aromatic rings.

So X-ray structure analysis was indicated. The analysis, which for practical reasons was performed on ( + )-mianserin hydrobromide, showed the conformation of Figure 1 a to be correct.

\section{EXPERIMENTAL DATA AND STRUCTURE DETERMINATION}

(+)-mianserin hydrobromide $\left(\mathrm{C}_{18} \mathrm{H}_{21} \mathrm{~N}_{2} \mathrm{Br}\right)$ forms small, colourless crystals from methanol. A small irregular shaped crystal $(0 \cdot 10 \times 0.22 \times$ $0.37 \mathrm{~mm}^{3}$ ) was selected for investigation.

Unit cell parameters and space-group determinations were carried out on a Stoe Weissenberg camera and a Nonius single-crystal diffractometer, with $\mathrm{Cu}-K_{\alpha}$ radiation. The unit cell was found to be orthorhombic, the space group being $P 2,2,2_{1}$. The unit-cell dimensions are: $a=1299(1) \mathrm{pm}, b=$ $1420(1) \mathrm{pm}, c=1002(1) \mathrm{pm}$.

On the basis of four molecules in the unit cell the density was calculated as $1.25 \mathrm{~g} . \mathrm{cm}^{-3}$, in agreement with the experimental observation (by flotation) of a density less than $1.59 \mathrm{g.} \mathrm{cm}^{-3}$.

Three-dimensional intensity data were recorded on a Nonius automatic single-crystal diffractometer, using $\mathrm{Cu}-K_{\alpha}$ radiation. About 1000 intensities were measured and corrected for Lorentz and

†The intensities can be obtained from the authors. polarization factors. $\dagger$ No absorption corrections were applied.

To determine the bromine-ion sites a threedimensional Patterson synthesis was calculated. Four possible bromine positions were found, but Fourier synthesis based on each possibility yielded a reasonable structure in only one case.

Subsequent least-squares refinement, based on most of the light atoms, and further Fourier synthesis revealed the positions of all atoms and indicated a pronounced anisotropy of the thermal motion of the Br-ions. Refinement with isotropic thermal parameters for the light atoms and anisotropic thermal parameters for the $\mathrm{Br}$-ion resulted in the fractional coordinates of Table 1 . However, in view of the fact that no absorption correction was applied, not much significance can be assigned to the thermal parameters, so they are not quoted. $\mathrm{H}$ atoms were not included in the refinement.

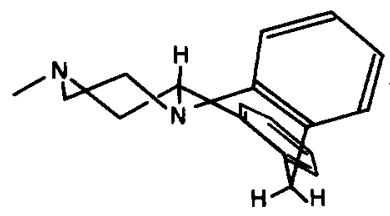

(a)

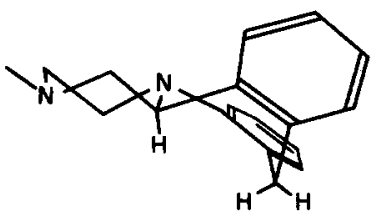

(b)

Fig 1. The two possible conformations of $(+)$-mianserin. (a) proves to be the correct one. 
Table 1. Fractional atomic coordinates of the absolute structure. Standard deviations (based on the least-squares analysis) are $\mathbf{0 . 0 0 1}$ to 0.002 .

\begin{tabular}{|c|c|c|c|}
\hline Atom & $x \cdot 10^{3}$ & $y \cdot 10^{3}$ & $z \cdot 10^{3}$ \\
\hline $\mathbf{B r}$ & 008 & 237 & 083 \\
\hline$C(1)$ & 155 & 552 & 209 \\
\hline $\mathbf{N}(2)$ & 041 & 569 & 222 \\
\hline C(3) & -009 & 481 & 243 \\
\hline C(4) & 030 & 438 & 370 \\
\hline $\mathrm{N}(5)$ & 137 & 418 & 350 \\
\hline$C(5 a)$ & 186 & 337 & 422 \\
\hline C(6) & 145 & 307 & 543 \\
\hline$C(7)$ & 187 & 226 & 609 \\
\hline$C(8)$ & 268 & 185 & 541 \\
\hline C(9) & 306 & 204 & 420 \\
\hline$C(9 a)$ & 263 & 294 & 359 \\
\hline$C(1 \theta)$ & 299 & 323 & 222 \\
\hline $\mathrm{C}(10 \mathrm{a})$ & 362 & 422 & 254 \\
\hline$C(11)$ & 465 & 425 & 229 \\
\hline $\mathrm{C}(12)$ & 517 & 502 & 264 \\
\hline C(13) & 481 & 574 & 334 \\
\hline$C(14)$ & 372 & 571 & 357 \\
\hline$C(14 a)$ & 318 & 495 & 315 \\
\hline$C(14 b)$ & 194 & 505 & 333 \\
\hline$C(15)$ & -002 & 621 & 101 \\
\hline
\end{tabular}

\section{Absolute configuration}

Use of the anomalous dispersion of X-radiation by the bromine ions allows the absolute configuration to be determined (Bijvoet's method $^{3}$ ). The atomic scattering factor becomes $f=f_{0}+\Delta f^{\prime}+$ $i \Delta f^{\prime \prime}$, where $f_{0}$ is the normal scattering factor, and $\Delta f^{\prime}$ and $\Delta f^{\prime \prime}$ are correction terms due to dispersion effects. For bromine ions and $\mathrm{Cu}-K_{\alpha}$ radiation $\Delta f^{\prime}=-0.9$ and $\Delta f^{\prime \prime}=1 \cdot 5{ }^{4}$

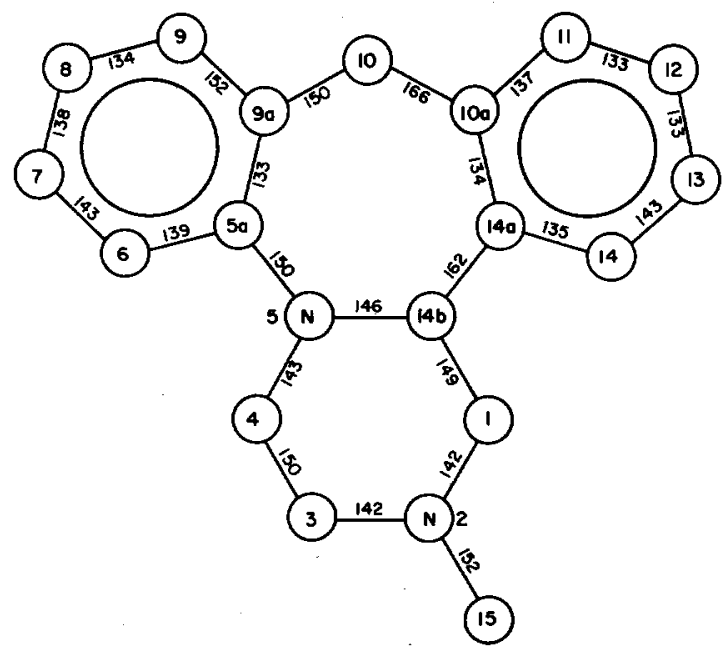

(o)

Fig 2a. Numbering of the atoms and the bond lengths in $\mathrm{pm}$ of $(+)$-mianserin. Standard deviations are $4 \mathrm{pm}$.

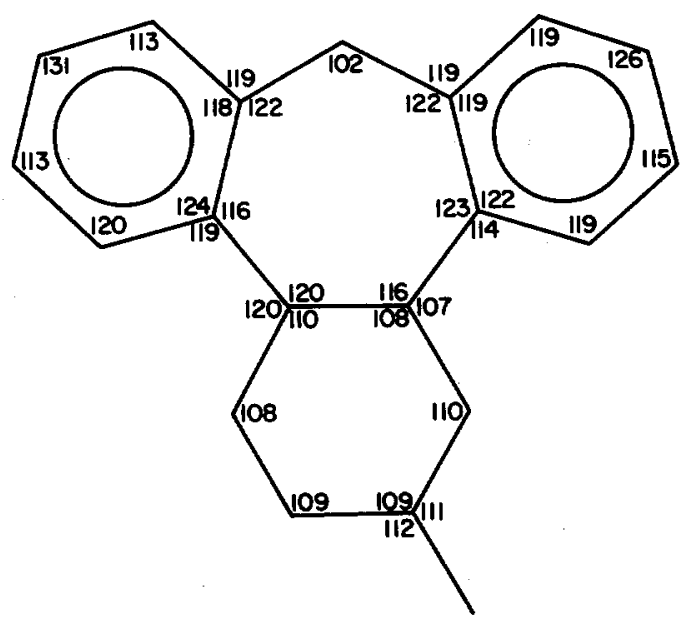

(b)

Fig 2b. The bond angles of (+)-mianserin. Standard deviations are $2^{\circ}$.

Those reflections where the contribution of the Br-ions is approximately $\pi / 2$ out of phase with the total scattering amplitude are the most sensitive to the anomalous scattering effect.

A number of $h k 1$ and $h k 2$ refiections obeying this condition was selected. The intensities of both these reflections and their counter reflections were measured on integrated Weissenberg photographs and the difference in intensity was compared with the difference calculated for the antipodes.

Of 14 pairs measured 10 showed significant differences in intensity, all of which were consistent with the structure shown in Fig 1a. As absorption of X-rays by the crystal is not negligible, lack of correction for absorption effects may result in differences of comparable magnitude. Another set of pairs was, therefore, selected for which anomalous scattering should not lead to a difference in intensity so that any observed differences can be attributed to absorption effects. None of the measured differences were significant.

\section{RESULTS}

The absolute chirality and conformation of the azepine ring in $(+)$-mianserin (1) found are shown in Fig 1a. The atomic coordinates for this structure, referred to a right-handed set of axes, are listed in Table 1 . The expected chair-like piperazino ring is shown to be present.

The distances of the $\mathbf{N}$-atoms of the piperazino ring from the neighbouring $\mathrm{Br}$-ions are:

$$
\begin{aligned}
& \mathrm{N}(2) \ldots \mathrm{Br}=314(2) \mathrm{pm} \\
& \mathrm{N}(5) \ldots \mathrm{Br}=407(2) \mathrm{pm} \ldots \mathrm{Br} \mathrm{p}^{\prime}=494(2) \mathrm{pm} \\
& \mathrm{N}(5) \ldots \ldots \mathrm{Br} r^{\prime}=495(2) \mathrm{pm}
\end{aligned}
$$



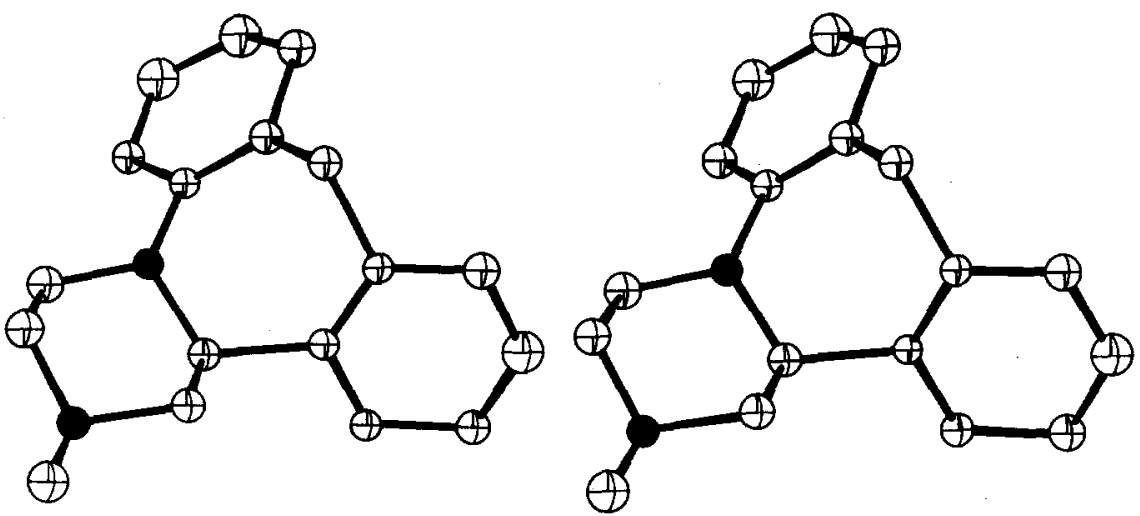

Fig 3. Stereoscopic view of (+)-mianserin.

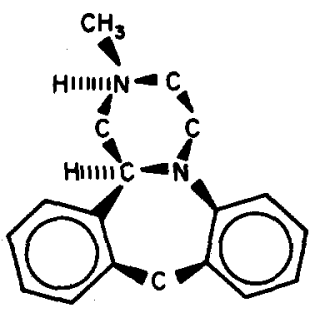

Fig 4. Conformation of ( + )-mianserin.

The numbering of the atoms and the bond lengths and angles are given in Figs $2 \mathrm{a}$ and $2 \mathrm{~b}$.

A stereoscopic view of the molecule is given in Fig 3, and a conventional diagram is shown in Fig 4.

\section{Discussion}

(+)-mianserin has an $(S)$-14b-configuration. The anti-relationship between the 14b-hydrogen and the lone pair of the 5-nitrogen is confirmed. The methylene bridge in the azepine ring is anti to the 14b-hydrogen. Although the position of the $H$ atoms has not been determined and, consequently, nothing definite can be said about the protonation of the piperazino ring, the location of the Br-ion makes it very likely that the $\mathrm{N}(2)$-atom has been protonated. The resulting hydrogenbond $\mathrm{N}(2)-\mathrm{H}$ ..... Br is about $100 \mathrm{pm}$ shorter than the sum of van der Waals radii.

van der Burg et al. ${ }^{1}$ suggested that the angle between the benzene rings would be comparable to that in cyproheptadine, which on the basis of measurements on Dreiding models was estimated as $120^{\circ}$. The present compound shows the expected correspondence of structural features, but the angle proves to be $106^{\circ} \pm 2 \cdot 5^{\circ}$.

Acknowledgements - Thanks are due to N. V. Organon in Oss for both their grant and the crystals, and to Dr. F. J. Zeelen for many helpful discussions.

\section{REFERENCES}

W. J. van der Burg, I. L. Bonta, J. Delobelle, C. Ramon and B. Vargaftig, J. Med. Chem. 13, 35 (1970)

${ }^{2} \mathrm{C}$. Ramon, Thèse d'Université, Paris (1969)

${ }^{3} G$. N. Ramanchandran, Advanced methods of Crystallography Vol. 67. Acad. Press (1964)

IInternational Tables for X-ray Crystallography 3, 214 (1962) 